\title{
CORRELATION EFFECTS IN THE BULK MODULUS AND EQUILIBRIUM LATTICE SPACING OF THE TRANSITION METALS
}

\author{
J. FRIEDEL
}

Physique des Solides (*)

Université Paris-Sud, 91405 Orsay, France

and

C. M. SAYERS

Department of Physics, Blackett Laboratory, Imperial College, London SW7 2AZ, England

(Reçu le 8 avril 1977, accepté le 26 mai 1977)

\begin{abstract}
Résumé. - Le rôle des corrélations dans le module de rigidité et le paramètre cristallin d'équilibre des métaux de transition est étudié par une méthode de perturbation reliée à l'approximation de Gutzwiller. On trouve qu'il décroît le module de rigidité et accroît le paramètre cristallin ; l'effet est maximum dans le milieu de la série $3 \mathrm{~d}$, en accord avec l'expérience.

Abstract. - The role of correlations in the bulk modulus and equilibrium lattice spacing of the transition metals is studied using a perturbation method related to Gutzwiller's approximation and is found to decrease the bulk modulus and increase the lattice spacing, the effect being greatest in the middle of the $3 \mathrm{~d}$ series in agreement with experiment.
\end{abstract}

As is seen in figure 1 the bulk modulus in the $4 \mathrm{~d}$ and $5 \mathrm{~d}$ transition metal series varies regularly with the

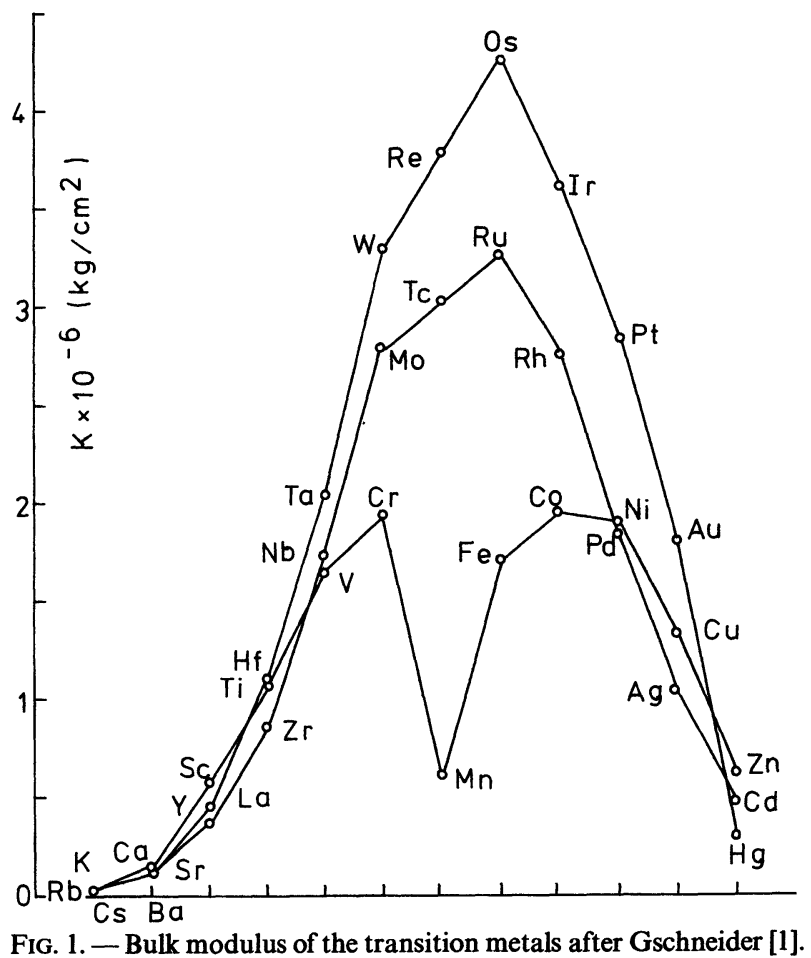

$\left({ }^{*}\right)$ L.A.du C.N.R.S. filling of the band with a maximum in the middle of the series [1] corresponding to the maximum cohesion found for half filled $d$ bands by Friedel [2]. In the 3d series, however, there is a marked deviation from this behaviour, the bulk modulus of $\mathrm{Cr}, \mathrm{Mn}, \mathrm{Fe}$ and $\mathrm{Co}$ being smaller than the values expected from the trend in the $4 \mathrm{~d}$ and $5 \mathrm{~d}$ series. A similar anomaly is seen in the equilibrium atomic volume of the $3 \mathrm{~d}$ series [1] shown in figure 2, the atomic volume of $\mathrm{Fe}$ and Co being larger than that of $\mathrm{Ni}$ in contrast to the behaviour in the $4 \mathrm{~d}$ and $5 \mathrm{~d}$ series where the atomic volume varies regularly with the filling of the $d$ band with a minimum corresponding to a maximum cohesion in the middle of the series. $\mathrm{Mn}$ is particularly notable for its large atomic volume and small bulk modulus, and has properties more like $\mathrm{Cu}$ in the same period with a full $\mathrm{d}$ band than $T c$ and $R e$ in the same group where the delectrons contribute strongly to the cohesion.

We have shown [3] that to second order in the electron-electron interaction energy $U$, the part of the $\mathrm{d}$ electron energy dependent on the bandwidth $W$ is

where

$$
E_{\mathrm{B}}=-A W-B / W
$$

$$
\begin{aligned}
& A=\frac{z}{20}(10-z) \\
& B=45\left[\frac{z}{10}\left(1-\frac{z}{10}\right) U\right]^{2}
\end{aligned}
$$




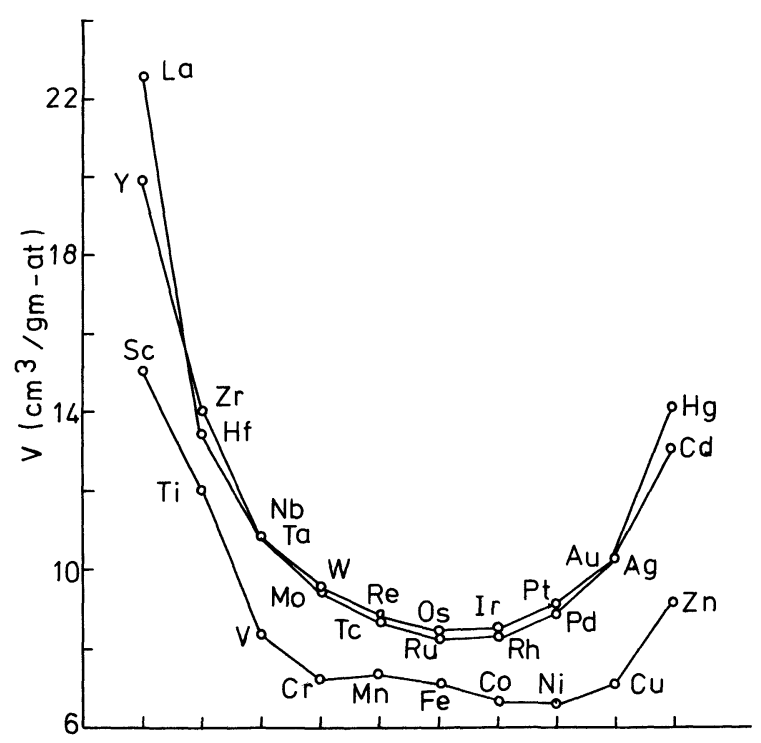

Fig. 2. - Atomic volume of the transition metals after Gschneider [1].

$z$ being the average number of electrons/atom in the $d$ band and where we have assumed five overlapping rectangular $\mathrm{d}$ bands for simplicity. The first term in (1) represents the band energy of the d electrons in the absence of correlations and gives good agreement with the measured cohesive energy in the $4 \mathrm{~d}$ and $5 \mathrm{~d}$ series [2]. The second term in (1) represents the first order contribution of correlations to the energy and reduces the electron interaction energy $9 z^{2} U / 20$ in the solid by preventing the electrons hopping on the same atom.

To study the equilibrium atomic volume and bulk modulus it is necessary to introduce a repulsive interaction between atoms, operative at short distances, to prevent the crystal collapsing under the cohesive forces, and following Ducastelle [4], who studied the elastic moduli and atomic radii in the transition metals neglecting correlation effects, we shall use the Born-Mayer form

$$
E_{R}=C \mathrm{e}^{-p R}
$$

where $R$ is the Wigner-Seitz radius, together with the approximation for the bandwidth

$$
W=W_{0} \mathrm{e}^{-q R} .
$$

Neglecting the correlation correction $B / W$ in (1) we obtain, upon minimising the energy, the equilibrium Wigner-Seitz radius

$$
R_{0}=\frac{1}{p-q} \ln \frac{p C}{q A W_{0}}
$$

as obtained by Ducastelle [4]. To first order in the correlation we then find for the relative change in the Wigner-Seitz radius due to correlations

$$
\propto \equiv \frac{R-R_{0}}{R_{0}} \approx \frac{9}{(p-q) R_{0}} \frac{z}{10}\left(1-\frac{z}{10}\right) \frac{U^{2}}{W^{2}} .
$$

The effect of correlations is thus seen to be a lattice expansion, the effect being greatest for a half filled $\mathrm{d}$ band (neglecting the dependence of $U, W, p R_{0}$ and $q R_{0}$ on $z$ ).

Taking the value of $p R_{0} \approx 6, q R_{0} \approx 3$ used by Ducastelle [4] and the average values $U \approx 3 \mathrm{eV}$, $W \approx 6 \mathrm{eV}$ deduced for the $3 \mathrm{~d}$ series [3] we find a $20 \%$ increase in the Wigner-Seitz radius in the middle of the $3 \mathrm{~d}$ series due to correlation effects which is rather larger than the observed anomaly. Inclusion of higher order terms in the perturbation expansion would act to reduce this correction.

The bulk modulus $K$ is given by

$$
K=V \frac{\mathrm{d}^{2} E}{\mathrm{~d} V^{2}}=\frac{R^{2}}{9 V} \frac{\mathrm{d}^{2} E}{\mathrm{~d} R^{2}}
$$

Neglecting correlation effects we find, following Ducastelle [4],

$$
K_{0}=\frac{q(p-q) R_{0}^{2}}{9 V} A W \approx \frac{A W}{V}
$$

using $p R_{0} \approx 6, q R_{0} \approx 3$, which varies parabolically with $z$ in good agreement with the measured values in the $4 \mathrm{~d}$ and $5 \mathrm{~d}$ series [4]. The relative change in the bulk modulus $\beta$ to first order in the correlation is then

$$
\begin{aligned}
\beta K_{0} & \equiv K-K_{0}=-\frac{q^{2} R^{2}}{V} 5\left[\frac{z}{10}\left(1-\frac{z}{10}\right)\right]^{2} \frac{U^{2}}{W} \\
& \approx-\frac{45}{V}\left[\frac{z}{10}\left(1-\frac{z}{10}\right)\right]^{2} \frac{U^{2}}{W}
\end{aligned}
$$

with $q R \approx 3$.

A very similar expression is obtained using Heine's [5] approximation $W \sim R^{-5}$ which gives upon differentiating the second term in (1) twice

$$
\beta K_{0}=-\frac{50}{V}\left[\frac{z}{10}\left(1-\frac{z}{10}\right)\right]^{2} \frac{U^{2}}{W} .
$$

Correlation is thus seen to reduce the bulk modulus, the reduction being greatest in the middle of the series. For $z=5, U=3 \mathrm{eV}$ and $W=6 \mathrm{eV}$, this reduction is of the order of $50-60 \%$ and is in good agreement with the magnitude of the anomaly observed in the $3 \mathrm{~d}$ series. Values deduced for $U$ and $W$ for the $4 \mathrm{~d}$ and $5 \mathrm{~d}$ series [3] lead to nearly negligible corrections $\alpha$ and $\beta$.

In a complete treatment the occurrence of magnetism in the second half of the $3 \mathrm{~d}$ series should also be included. We shall not discuss here the antiferromagnetism of $\mathrm{Cr}$ and $\mathrm{Mn}$ but only the ferromagnetism of $\mathrm{Fe}, \mathrm{Co}$ and $\mathrm{Ni}$. We have shown [3] that in the ferromagnetic state with $(z+\mu) / 2$ electrons per atom 
having spin $\uparrow$ and $(z-\mu) / 2$ having spin $\downarrow$ that $A$ and $B$ transform to become

$$
\begin{gathered}
A=\frac{z}{20}(10-z)-\frac{\mu^{2}}{20} \\
B=45\left[\frac{z}{10}\left(1-\frac{z}{10}\right) U\right]^{2} \\
+\left[-1-0.14 z(10-z)+0.09 \mu^{2}\right] U^{2} \frac{\mu^{2}}{20} .
\end{gathered}
$$

Eq. (11) has been derived for the case of five overlapping rectangular bands [3] and holds for all values of $\mu^{2}$. The term in $\mu^{4}$ has been shown [3] to be important in determining the stability of ferromagnetism and arises from the change in phase space available for electron-electron scattering upon magnetization. This term is known to be necessary in the itinerant model of ferromagnetism in order to obtain agreement with the observed magnetization versus temperature curves [6].

Using (11) the effect of magnetization on the Wigner-Seitz radius and bulk modulus may be investigated. Neglecting the correlation correction $B / W, R_{0}$ transforms such that

$$
\frac{\delta R_{0}}{R_{0}}=-\frac{1}{(p-q) R_{0}} \ln \left[\frac{z(10-z)-\mu^{2}}{z(10-z)}\right]
$$

where, for a strong ferromagnet at the end of the series such as $\mathrm{Co}$ or $\mathrm{Ni}, \mu=(10-z)$.

Similarly neglecting correlation effects the change in the bulk modulus due to magnetization is

$$
\frac{\delta K_{0}}{K_{0}}=-\frac{\mu^{2}}{z(10-z)} .
$$

In addition magnetization reduces the correlation corrections $\alpha$ and $\beta$ to $R$ and $K$ by reducing the number of interactions between electrons of opposite spin. Table I compares the correlation corrections $\alpha_{\mu^{2}}$ and $\beta_{\mu^{2}}$ in the magnetic state with those in the nonmagnetic state $\alpha_{\mathrm{NM}}$ and $\beta_{\mathrm{NM}}$ given by (6) and (9), and with the corrections to $R_{0}$ and $K_{0}$ due to magnetization in the absence of correlations given by (12) and (13). Both magnetization and correlation act to decrease the bulk modulus and increase the Wigner-Seitz radius due to the transfer of electrons into orbitals of high kinetic energy, the effect of magnetization being rather smaller than that of correlation.

\begin{tabular}{|c|c|c|c|}
\hline & - $\mathrm{Fe}$ & Co & $\mathrm{Ni}$ \\
\hline & - & - & - \\
\hline$z$ & 7.1 & 8.3 & 9.4 \\
\hline$\mu$ & 2.2 & 1.7 & 0.6 \\
\hline$\alpha_{\mathrm{NM}}$ & 0.154 & 0.106 & 0.042 \\
\hline$\alpha_{\mu^{2}}$ & 0.114 & 0.075 & 0.035 \\
\hline$\delta R_{0} / R_{0}$ & 0.089 & 0.076 & 0.022 \\
\hline$\beta_{\mathrm{NM}}$ & -0.463 & -0.317 & -0.127 \\
\hline$\beta_{\mu} 2$ & -0.341 & -0.224 & -0.106 \\
\hline$\delta K_{0} / K_{0}$ & -0.235 & -0.205 & -0.064 \\
\hline
\end{tabular}

\section{TABLE I}

Comparison of the effect of correlation and ferromagnetization on the Wigner-Seitz radius and bulk modulus of $\mathrm{Fe}, \mathrm{Co}$ and $\mathrm{Ni}$.

In conclusion, correlation effects are found to have their maximum effect for a half filled $\mathrm{d}$ band and are responsible to a large extent [7] for the anomaly in the behaviour of the bulk modulus and Wigner-Seitz radius observed in the $3 \mathrm{~d}$ transition metal series.

Acknowledgments. - One of us (C.M.S.) wishes to thank Professor N. H. March for several useful discussions and the SRC for the award of a Postdoctoral Research Fellowship.

\section{References}

[1] Gschneider, K. A., Solid State Phys. 16 (1964) 275.

[2] Friedel, J., In Physics of Metals I, Electrons, Ed. J. Ziman (Cambridge University Press) 1969.

[3] Friedel, J. and SaYers, C. M., J. Physique (1977) under press.

[4] Ducastelle, F., J. Physique 31 (1970) 1055.
[5] Heine, V., Phys. Rev. 153 (1967) 673.

[6] Hunt, K. L., Proc. R. Soc. A 216 (1953) 103.

[7] Cf. JanaK, J. F. and Williams, A. R., Phys. Rev. B 14 (1976) 4199 , for another treatment of the magnetic term. 